\title{
GSR extinction following differential reinforcement
}

ROBERT S. BEECROFT OKLAHOMA STATE UNIVERSITY

\begin{abstract}
Extinction was studied for 24 trials following differential GSR conditioning. The discrimination did not persist in extinction. This may reflect a sudden collapse of discrimination with the onset of extinction or failure to establish a stable discrimination during acquisition training.
\end{abstract}

\section{Problem}

Little information is available concerning extinction following differential classical conditioning. Hilgard et al (1938) ran an abbreviated extinction series following two days of differential eyelid conditioning; their results were inconclusive. The present study was undertaken with the more slowly extinguishing conditioned GSR。

\section{Method}

The positive CS (CS+), an $800 \mathrm{cps}$ tone, was reinforced eight times. Then, 24 differential reinforcement trials were given in which $\mathrm{CS}+$ was reinforced eight more times and the negative CS (CS-), a $1200 \mathrm{cps}$ tone, was presented 16 times without reinforcement. During the differential reinforcement series $\mathrm{CS}+$ was never presented on two successive trials and CS- did not occur on more than three successive trials. After differential training there were 24 extinction trials equally divided between the CSs. The CS duration was $500 \mathrm{msec}$. with a $450 \mathrm{msec}$. CS-UCS interval for CS+during reinforced training. The intertrial interval varied from 25 to $35 \mathrm{sec}$. Recording electrodes were on the palm and back of S's left hand; a $50 \mathrm{msec}$. DC shock was delivered to the right middle finger. The response measure was the square root of conductance change. The analysis is based on $24 \mathrm{Ss}$.

\section{Results}

Data for nonreinforced trials are shown in Fig. 1. The curve on the left is for CS- during the differential reinforcement series. After the temporary decrement on the second and third CS- trials, there was a gradual extinction of the generalized GSR to CS-. When CS+ was first tested after 16 reinforcements (isolated dot), the mean GSR was 0.25 greater than the mean GSR to CS- on the immediately preceding trial, though this difference was not statistically significant, $t(23)=0.67$. Following the first nonreinforcement of CS+, the response level dropped sharply for both CSs. After the initial drop there was no difference in the extinction curves for the CSs (mean difference was -0.02) although extinction proceeded at a slow pace.

\section{Discussion}

Once reinforcement is discontinued the GSR to CSis maintained as well as the GSR to CS+. The lack of a differential in extinction could either be due to a failure to establish a satisfactory conditioned discrimination

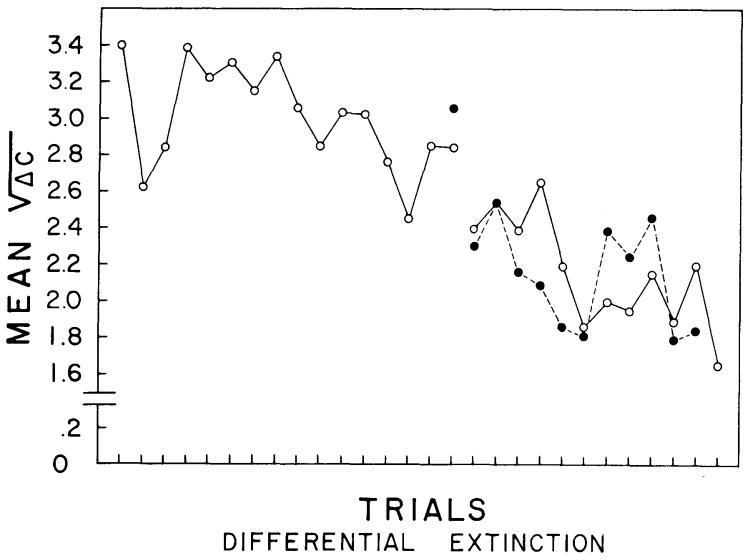

Fig. 1. Mean square root of conductance change during differential reinforcement and extinction for CS- (open dots) and CS+ (solid dots).

prior to extinction or to a rapid collapse of the discrimination once $\mathrm{CS}+$ is nonreinforced. The absolute level of GSR discrimination is low. Using the RestleBeecroft (1955) index of relative differentiation (IRD), the present data yield a mean IRD of .07 at the end of acquisition training. The mean IRD for the extinction series was .00 . Application of the formula to the 16 reinforcement, $500 \mathrm{msec}$. means obtained by Kimmel \& Pennypacker (1963) also gives an IRD of .07. These IRD values are well below those observed in eyelid conditioning (cf. Restle \& Beecroft, 1955). Campbell (1936), working in Hilgard's laboratory, also experienced difficulty in differential GSR conditioning with visual stimuli which produced good conditioned eyelid discrimination. Some technique for improving conditioned GSR discrimination is needed since failure to observe differential extinction performance could easily be due to lack of a stable discrimination.

\section{References}

Campbell, R. K. Some difficulties in conditioning galvanic skin responses. Psychol. Bull., 1936, 33, 618-619.

Hilgard, E. R., Campbell, R. K., \& Sears, W. N. Conditioned discrimination: the effect of knowledge of stimulus relationships. Amer. J. Psychol., 1938, 51, 498-506.

Kimmel, H. D., \& Pennypacker, H. S. Differential GSR conditioning as a function of the CS-UCS interval. J. exp. Psychol., 1963, $65,559-563$.

Restle, F., \& Beecroft, R. S. Anxiety, stimulus generalization, and differential conditioning: a comparison of two theories. Psychol. Rev., 1955, 62, 433-437.

Note

1. This research was conducted at Furman University and supported by NSF grant 16027. The author wishes to thank Brenda Avery and Angie Hutto for their assistance in collecting and analyzing the data. 